\title{
The incidence of clinical malaria detected by active case detection in children in Ifakara, southern Tanzania
}

\author{
D. M. Schellenberg ${ }^{1,2}$, J. J. Aponte ${ }^{1}$, E. A. Kahigwa ${ }^{2,3}$, H. Mshinda ${ }^{2}$, M. Tanner ${ }^{4}$, C. Menendez ${ }^{1}$ and P. L. \\ Alonso ${ }^{1}{ }^{1}$ Centro de Salud International, Institut d'Investigacions Biomedicas August Pi $i$ Sunyer (IDIBAPS), Hospital \\ Clinic, Villarroel 170, 08036 Barcelona, Spain; ${ }^{2}$ Ifakara Health Research and Development Centre, P.O. Box 53, Ifakara, \\ Tanzania; ${ }^{3}$ St Francis Designated District Hospital, Ministry of Health, Dar es Salaam, Tanzania; ${ }^{4}$ Swiss Tropical Institute, \\ Socinstrasse 57, Postfach CH 4002, Basle, Switzerland
}

\begin{abstract}
Between July 2000 and June 2001, we used weekly active case detection (ACD) of clinical malaria episodes in 618 children aged $<5$ years to describe the epidemiology of malaria in Ifakara, southern Tanzania. Plasmodium falciparum-positive blood slides prepared from children with axillary temperature $\geqslant 37.5^{\circ} \mathrm{C}$ were used to define clinical malaria and a rolling cross-sectional survey documented the prevalences of parasitaemia and anaemia. A random subsample of children was visited daily for 1 month at the end of the study to assess the effect of more frequent visits on estimated incidence rates. Only 50 $(8 \%)$ children had 1 or more episodes of clinical malaria during the year, an overall incidence of 0.275 episodes $/ 100$ child-weeks-at-risk, with no age dependence. The maximum parasite prevalence of $25 \%$ was reached in children aged 4 years. The incidence of illness was significantly lower in children visited daily than in those visited weekly, suggesting a marked effect of frequent visits on estimated incidence rates. We conclude that the age pattern of malaria detected through ACD is a more robust epidemiological indicator than absolute incidence rate estimates and that, in contrast to the surrounding area, Ifakara town is subject to only moderate perennial malaria transmission.
\end{abstract}

Keywords: malaria, Plasmodium falciparum, epidemiology, active case detection, Tanzania

\section{Introduction}

A clear picture of the malaria epidemiology of a study site is required to assess the potential wider applicability of interventions assessed there. The entomological dimension is most frequently summarized as the number of infectious bites an individual receives per unit time, the entomological inoculation rate (EIR). Agespecific parasite, anaemia and spleen prevalences contribute to the epidemiological picture, as do hospital-based studies describing the clinical presentation of Plasmodium falciparum disease. The incidence of clinical disease may be documented by active or passive case detection. Active case detection (ACD), where people are visited regularly at home, describes the age pattern and temporal variations of disease without the influence of health-seeking behaviour. Passive case detection (PCD) depends on documenting episodes in children presenting to the health facility and may generate information of greater relevance from a health services perspective. In the assessment of a randomly assigned intervention either approach should generate useful efficacy estimates. However, as incidence rates generated by ACD are not comparable with those generated by PCD, the value of these measures in the description of the malaria epidemiology of different sites is limited. Possibly the most valuable information relates to the age pattern of clinical disease and death, as it not only reflects the intensity of transmission but also indicates the age group against which preventive interventions need to be targeted.

Ifakara town, situated in the Kilombero district of southern Tanzania, has been the site of a series of malaria and anaemia intervention trials. The EIR in several villages surrounding the town has been estimated at more than 200 infective bites per person per year (Tanner et al., 1986; Lyimo, 1993; Smith et al., 1993; Charlwood et al., 1998) and an analysis of the age pattern of paediatric malaria admission and death in the district hospital showed a pattern typical of an area with intense malaria transmission (Schellenberg et al., 1999). However, over recent years it has become

Address for correspondence: David Schellenberg, Centro de Salud International, Institut d'Investigacions Biomedicas August $\mathrm{Pi}$ i Sunyer (IDIBAPS), Hospital Clinic, Villarroel 170,08036 Barcelona, Spain; phone +3432275706 , fax +34 34515272 , e-mail dmschellenberg@aol.com increasingly clear that the pattern of malaria in children from Ifakara town itself is different from that in the surrounding area. This paper uses a 1-year ACD study to describe the epidemiology of malaria in Ifakara town and discusses various methodological aspects of ACD.

\section{Materials and Methods \\ Study area}

The map (Fig. 1) includes the study area and indicates the homes of participating children. Ifakara is 1 of the 5 divisions of Kilombero district, in the Morogoro region of southern Tanzania. Ifakara division is split into 5 wards, 3 of which (Ifakara, Kibaoni and Lumemo) contribute to the study area described in this paper and have been used in previous studies (Menendez et al., 1997; Hatz et al., 1998; Acosta et al., 1999; Schellenberg et al., 2001, 2002). All villages in Ifakara ward (Ifakara Mjini, Lipangalala, Viwanja Sitini and Mlabani), Mbasa village from Kibaoni ward and Lumemo, Michenga, Mahutanga and Kiningina villages from Lumemo ward contribute to a total population of approximately 86000 in the area. The 9 villages were selected as they are situated within $\sim 6 \mathrm{~km}$ of the St Francis Designated District Hospital (SFDDH; $36^{\circ} 40^{\prime}$ $\mathrm{E}, 8^{\circ} 9^{\prime} \mathrm{S}$ ), ensuring good access to the PCD system based there. The area is subject to a long rainy season from March to June, followed by dry and dusty weather until November-December when light rains return.

Ifakara town is more heterogeneous than the surrounding villages but can be split into 2 main parts: a semi-urban centre with a population of $\sim 50000$ (Ifakara Mjini, Viwanja Sitini and Mlabani) and the remaining, more rural, villages with a population of $\sim 35000$. An unpaved road runs through the central area and is flanked by 2 smaller parallel streets, all lined with brick-built shops and bars. Some of these buildings have an electricity supply but few have piped water. Beyond these streets lie the densely populated residential areas which gradually merge with the more rural areas, similar to those involved in the Ifakara Health Research and Development Centre (IHRDC) Demographic Surveillance System (DSS), described in detail elsewhere (Armstrong Schellenberg et al., 2001). Houses in this more rural area are typically made with mud or burnt brick walls and grass or corrugated iron roofs. Electricity is supplied to fewer houses and most people collect water from either the nearby Lumemo 


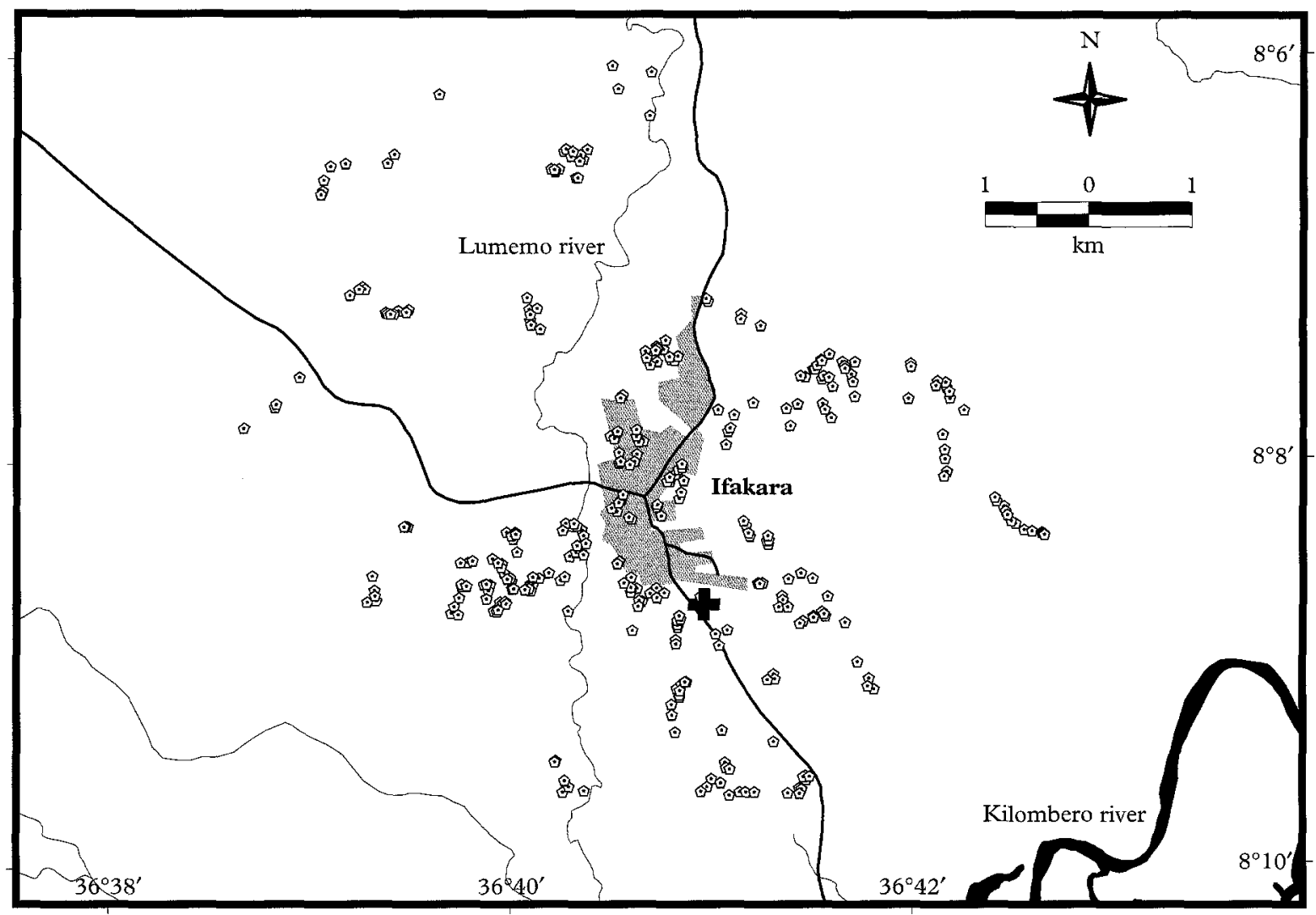

- Household of children included in the study

Saint Francis Designated District Hospital

Urban area $\quad$ River $\longrightarrow$ Road

Fig. 1. Map of the Ifakara area, southern Tanzania, indicating the locations of study children's households.

river or shallow wells. Thick vegetation and abundant mosquito breeding sites are found between dwellings in these residential areas.

\section{Selection and recruitment of study participants}

Tanzanian households are arranged into 'cells' of about 10 (range 7-23), with one of the residents named as balozi, or the 10-cell leader. An updated list of all balozis in the study area was obtained and 110 selected at random by a computer program. A random three-quarter sample of each selected balozi's households was then generated by computer and written informed consent sought to enrol all children aged $<5$ years in each of these households. This approach was anticipated to provide approximately 100 children in each 1-year age group. Children who were not permanent residents of Ifakara or who were involved in other studies were not eligible to participate. To ensure that children in the youngest age groups were adequately represented during the whole of the study up to 8 children aged $<1$ month were recruited monthly from households in the original sampling scheme.

A recruitment form, completed at the child's home, documented demographic and socio-economic information. Data were also collected on factors which might be associated with the risk of malaria, such as use of a mosquito net, home ownership (whether owneroccupier or rented), type of roof, floor and walls, ownership of chickens, radio or bicycle, and the area of land cultivated. Each house was geolocated using a Garmin GPS 12 (Garmin International, Olathe, KS, USA) which enabled calculation of the distance between the house and SFDDH using the Haversine formula (Sinnot, 1984). Each child was left with an identity card and the carer was given verbal and written instructions detailing how to access project clinical staff in the event of illness.

\section{Follow-up}

Between July 2000 and June 2001, home visits were repeated weekly, before 11:00, in order to minimize biases due to the diurnal variation in body temperature (Armstrong Schellenberg et al., 1994). At each visit a prelabelled standard morbidity questionnaire was completed. Two blood slides were prepared from a fingerprick if the axillary temperature was $\geqslant 37.5^{\circ} \mathrm{C}$ or there was a history of fever in the preceding $24 \mathrm{~h}$. Carers were informed of blood slide results within $4 \mathrm{~h}$ and antimalarial treatment taken to them at home when necessary. A substudy investigated the effect on the detected incidence of fever of more frequent home visits. At the end of 1 year's complete follow-up, 1 in 7 children was randomly selected from the cohort and visited daily for 1 month, while the remainder of the cohort continued with weekly visits.

In the event of any illness, mothers were encouraged to take their child to the IHRDC-employed clinical officers operating the PCD system at SFDDH. De- 
scribed in detail elsewhere (Schellenberg et al., 1999), this system operates $24 \mathrm{~h}$ a day, every day of the year, to document illness episodes in study children. The same criteria for blood slide collection were used as at ACD visits. Free drug treatment was made available as required.

Each week, 10 children were randomly selected (2 from each 1-year age group) and a blood slide and packed cell volume (PCV) sample collected, regardless of fever. This rolling cross-sectional survey allowed assessment of temporal variations and the age dependence of asymptomatic parasitaemia and anaemia. Children with a PCV $<25 \%$ were supplied with the standard anaemia treatment $(14 \mathrm{~d}$ of ferrous sulphate and a treatment dose of sulphadoxine-pyrimethamine [SP]) and malaria episodes were treated with SP, which remains effective in the area (Schellenberg et al., 2002). The study was approved by the IHRDC and national Medical Research Co-ordinating Committee ethical committees.

\section{Quality control of field activities}

Twenty randomly selected households were revisited by the field supervisor each week. He checked that fieldworkers had visited the household and whether children had reported a fever or had a blood sample collected. This information was checked against the original fieldworker's report. The field supervisor also accompanied fieldworkers on a small number of visits to ensure that all procedures were carried out correctly and sensitively.

\section{Laboratory procedures}

Laboratory tests were performed at IHRDC. Standard blood slide reading and storage procedures were followed, described in detail elsewhere (Alonso et al., 1994). In brief, all slides were read twice and a computerized comparison routine used to identify slides with discrepant results which were then read a third time. The PCVs were measured in microcapillary tubes using a Hawkesley haematocrit reader after centrifugation. Red cell pellets remaining after PCV reading of samples collected at the rolling cross-sectional survey were stored at $4^{\circ} \mathrm{C}$ for up to 1 week before haemoglobin electrophoresis on cellulose acetate plates.

\section{Data management and analysis}

Data were double-entered into Microsoft FoxPro version 2.6 (Microsoft Corp., Seattle, WA, USA) databases and weekly completeness and internal integrity checks performed. Analysis was done using Stata version 7 (Stata Corp., College Station, TX, USA) according to a pre-agreed analytical plan. Clinical malaria was defined as a measured axillary temperature $\geqslant 37.5^{\circ} \mathrm{C}$ in the presence of asexual $P$. falciparum parasitaemia. Episodes of clinical malaria detected at ACD visits, or at a PCD visit which occurred on the day of a scheduled ACD visit, were included in the analysis. A child did not contribute to the numerator or denominator for $28 \mathrm{~d}$ after a clinical malaria episode. Unless otherwise indicated, each weekly visit was considered as $7 \mathrm{~d}$ time at risk. The incidence of clinical malaria was compared between the different age groups using Poisson regression taking into account the 2-stage sampling procedure.

\section{Results}

A total of 649 children were invited to participate, of whom $618(95 \%)$ gave informed consent. This included 550 children aged $<5$ years recruited in July 2000 and a further 68 children recruited shortly after birth during the course of the year. Fifteen children $(2 \%)$ died (6 [40\%] in infancy) and a further $15(2 \%)$ withdrew. Basic descriptive features of the cohort are summarized in Table 1 and the age breakdown at recruitment is presented in Table 2 . During the main
Table 1. Characteristics of 618 study children, Ifakara, Tanzania

\begin{tabular}{lc}
\hline & $n(\%)$ \\
\hline Male gender & $295(47.7)$ \\
Owner-occupier of house & $529(85.6)$ \\
Roof type & $255(41.3)$ \\
$\quad$ Grass & $363(58.7)$ \\
$\quad$ Corrugated iron & \\
Floor type & $494(79.9)$ \\
Earth & $124(20.1)$ \\
Cement & $150(24.3)$ \\
Wall type & $29(4.7)$ \\
Mud & $439(71.0)$ \\
Mud bricks & $29(4.7)$ \\
Burnt mud bricks & $198(32.0)$ \\
Electricity supply to house & $166(26.9)$ \\
Assets & \\
Bicycle & $223(36.1)$ \\
Radio & $15(2.4)$ \\
Chicken & $6(1.0)$ \\
Cow and/or pig & $6(1.0)$ \\
Television & $376(60.8)$ \\
Refrigerator & \\
Mosquito net use & $37(9.8)$ \\
Last insecticide treatment $(n=376)$ & $22(5.8)$ \\
$<6$ months ago & $30(8.0)$ \\
$7-11$ months ago & $287(76.3)$ \\
₹12 months ago & \\
Never & \\
\hline
\end{tabular}

${ }^{\mathrm{a}}$ One or more of each.

study, 27567 home visits were made and the child was present at 19069 of these. Data were available from PCD at the time of a scheduled ACD visit for 192 additional contacts. Hence, data were available from $19261(70 \%)$ scheduled contacts and the following analyses are based on these data.

During the course of the year fever was reported on $302(1.6 \%)$ occasions and a raised body temperature documented $171(0.9 \%)$ times. Of these measured fevers, almost one-third $(n=53)$ were detected through $P C D$ at the time of a scheduled $A C D$ visit. The incidence of measured fever was strongly agedependent (Fig. 2), being highest in infants (1.7 episodes $/ 100$ child-weeks-at-risk [CWAR], 95\% CI 1.3-2.2), and falling during the second year of life before reaching a plateau in children aged 2 years (0.7 episodes $/ 100$ CWAR, 95\% CI 0.48-0.93) $(P<$ $0.001)$. There were also marked changes in the incidence of measured fever at different times of year (Fig. 3 ), with incidence rates of around 1.1 episodes $/ 100$ CWAR during July-December, and a peak of 1.8 episodes $/ 100$ CWAR in January falling gradually to a minimum of 0.1 episodes $/ 100$ CWAR in April $\left(\chi^{2}\right.$ test for heterogeneity $=43.9, P<0.001)$. The incidence of measured fever was not associated with use of a mosquito net $(P=0.9)$ but increased significantly with increasing distance from SFDDH $(P=0.001$ ) (data not shown).

Twenty-seven percent $(87 / 328)$ of the blood samples collected from sick children during weekly visits were positive for asexual $P$. falciparum. Fifty ( $8 \%$ ) children fulfilled the criteria for clinical malaria at least once, and $3(0.5 \%)$ children had 2 episodes during the year. Sixteen of the 53 cases $(30 \%)$ were detected by PCD. The geometric mean parasite densities of malaria episodes detected by PCD and ACD were 5513 $(\mathrm{SD}=13481)$ and $8406(\mathrm{SD}=19598)$, respectively $(P=0.554)$. The overall crude incidence rate of clinical malaria in children aged $<5$ years was 0.275 episodes $/ 100 \mathrm{CWAR}$ and did not vary significantly in the different 1-year age groups (Table 2). The inci- 
Table 2. Age-specific incidence of clinical malaria in study children, Ifakara, Tanzania

\begin{tabular}{lcccc}
\hline $\begin{array}{l}\text { Age } \\
\text { (years) }\end{array}$ & $n(\%)$ & $\begin{array}{c}\text { No. of } \\
\text { cases }\end{array}$ & $\begin{array}{c}\text { Child-weeks- } \\
\text { at-risk }\end{array}$ & $\begin{array}{c}\text { Incidence rate (95\% CI) } \\
\text { of clinical malaria }\end{array}$ \\
\hline 0 & $191(31)$ & 9 & 3619 & $0.249(0.129-0.478)$ \\
1 & $115(19)$ & 10 & 2981 & $0.335(0.18-0.623)$ \\
2 & $158(26)$ & 10 & 5095 & $0.196(0.106-0.365)$ \\
3 & $107(17)$ & 13 & 4875 & $0.267(0.155-0.459)$ \\
4 & $47(8)$ & 11 & 2691 & $0.409(0.226-0.738)$ \\
Total & & 53 & 19261 & $0.275(0.210-0.360)$ \\
\hline
\end{tabular}

${ }^{a}$ Per 100 child-weeks-at-risk

$\chi^{2}$ test for heterogeneity $(4$ d.f. $)=3.40, P=0.494$

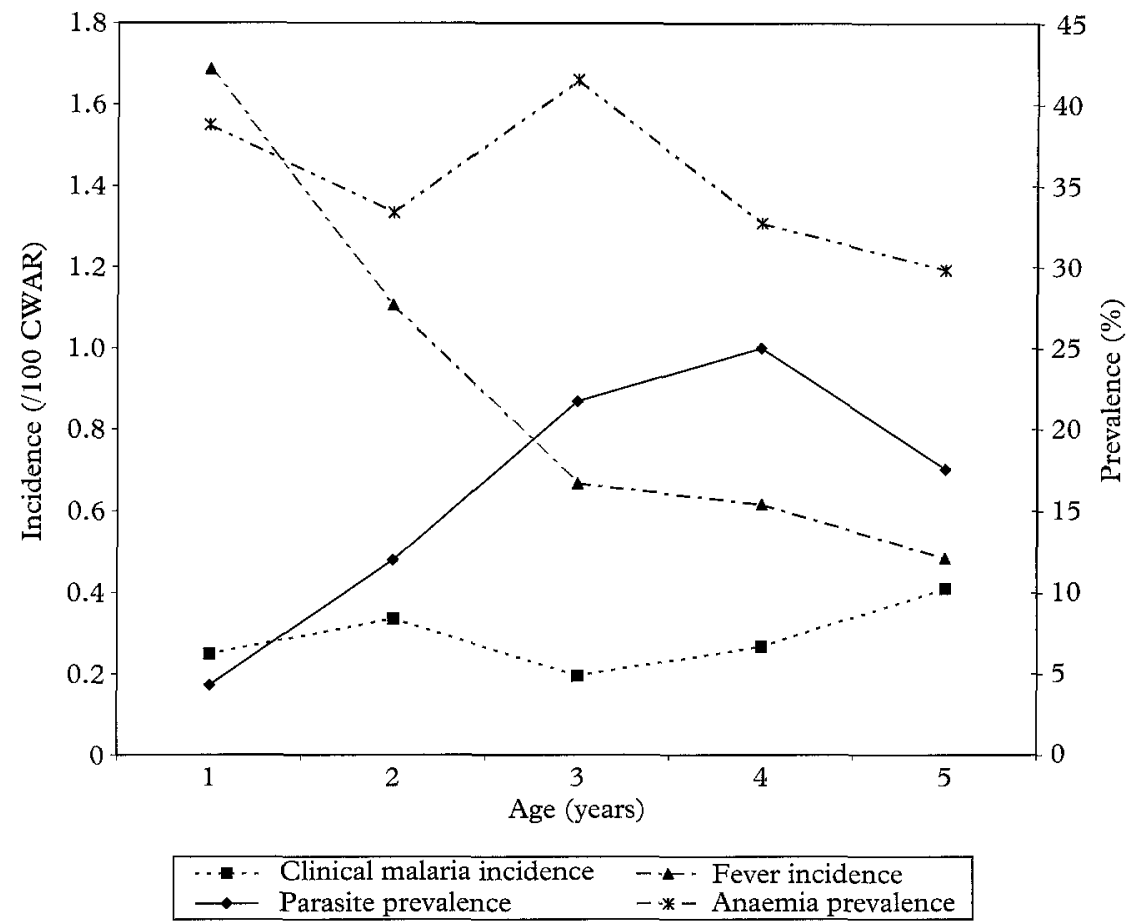

Fig. 2. Relationship between age and incidences of clinical malaria and fever, and prevalences of parasitaemia and fever, in study children, Ifakara, Tanzania. Incidence was measured per 100 child-weeks-at-risk.

dence of clinical malaria was significantly associated with month of the year, reaching a maximum in January (Table 3), and was also significantly higher in children not sleeping under mosquito nets and in those living further away from the hospital. These temporal and geographical effects remained significant after Poisson multiple regression. Children sleeping in houses with iron roofs tended to have less malaria than those sleeping under grass roofs (Table 3 ).

A total of 435 visits were conducted as part of the rolling cross-sectional survey. A history of fever was reported in $4.1 \%(n=18)$ of children and $1.1 \%(n=5)$ had a measured pyrexia. Although $17 \%(n=72)$ of samples were positive for $P$. falciparum, none of these was from a child with measured fever and hence no child fulfilled the case definition for clinical malaria on cross-sectional survey. There was marked age dependence of parasite prevalence (Fig. 2), with low levels in the first 2 years of life and a peak prevalence of $25 \%$ in 4 -year-old children $\left(\chi^{2}=18.7, P=0.001\right)$. The variation with time in parasite prevalence did not reach statistical significance $\left(\chi^{2}=14.4, P=0.21\right)$. Over the course of the year, $35.9 \%(156 / 435)$ of children in the rolling cross-sectional survey were anaemic. Most of the anaemia was mild, only $3 \%(12 / 435)$ having a PCV $<25 \%$ and none having a PCV $<15 \%$.

Figure 2 shows that, as the incidence of fever decreases, the prevalence of asymptomatic parasitaemia increases. There was no clear variation with age of incidence of clinical malaria or the prevalence of anaemia. The marked temporal variation in the incidence of fever is shown in Fig. 3, and mirrors the changes in the incidence of clinical malaria. Both were maximal 1 month after the rainy season started. The highest parasite prevalence was observed 2 months after the peak in clinical malaria and fever. There was also marked temporal variation in the prevalence of anaemia $\left(\chi^{2}=31.2, P=0.001\right)$, the lowest prevalence occurring 1 month after the highest incidence of clinical malaria and one of the highest anaemia prevalences arising a month after the peak prevalence of asymptomatic parasitaemia.

The children selected for daily visits were similar to the remainder of the cohort in terms of age, distance between their homes and the hospital and reported use of a mosquito net (data not shown). During the 28-d period of daily follow-up the incidence rates of reported fever and measured pyrexia in children visited daily 


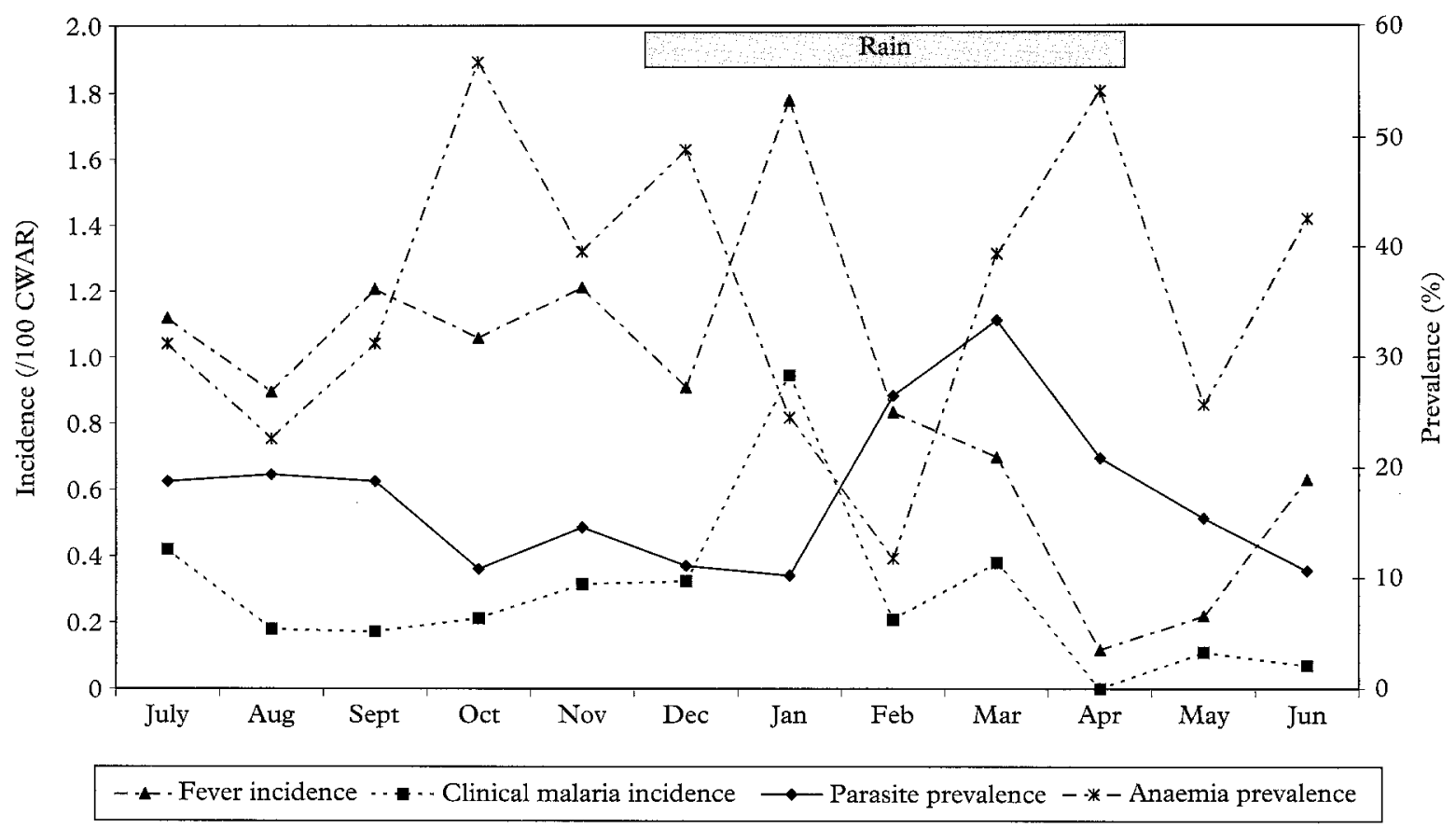

Fig. 3. Incidences of fever and clinical malaria, and prevalences of parasistaemia and anaemia, by month, in study children, Ifakara, southern Tanzania. Incidence was measured per 100 child-weeks-at-risk.

Table 3. Associations $(P<0.1)$ with incidence of clinical malaria in study children, Ifakara, Tanzania

\begin{tabular}{lccc}
\hline Variable & $\begin{array}{c}\text { No. of } \\
\text { cases }\end{array}$ & $\begin{array}{c}\text { Child-weeks- } \\
\text { at-risk }\end{array}$ & $\begin{array}{c}\text { Incidence rate }(95 \% \mathrm{CI}) \\
\text { of clinical malaria }\end{array}$ \\
\hline $\begin{array}{l}\text { Month }(P<0.01) \\
\text { July }\end{array}$ & 3 & 715 & $0.420(0.135-1.301)$ \\
August & 3 & 1678 & $0.179(0.058-0.554)$ \\
September & 3 & 1740 & $0.172(0.056-0.535)$ \\
October & 4 & 1891 & $0.212(0.079-0.564)$ \\
November & 6 & 1899 & $0.316(0.142-0.703)$ \\
December & 5 & 1542 & $0.324(0.135-0.779)$ \\
January & 17 & 1799 & $0.945(0.587-1.52)$ \\
February & 3 & 1442 & $0.208(0.067-0.645)$ \\
March & 6 & 1578 & $0.380(0.171-0.846)$ \\
April & 0 & 1722 & 0.000 \\
May & 2 & 1825 & $0.110(0.027-0.438)$ \\
June & 1 & 1430 & $0.070(0.01-0.496)$ \\
Distance from hospital $(\mathrm{km})$ & $(P=0.002)$ & 6453 & $0.155(0.083-0.288)$ \\
0.3-1.39 & 10 & 5908 & $0.203(0.115-0.358)$ \\
1.39-2.2 & 12 & 6900 & $0.449(0.316-0.639)$ \\
2.2-5.2 & 31 & 11595 & $0.198(0.132-0.299)$ \\
Mosquito net use $(P=0.012)$ & 23 & 7666 & $0.391(0.274-0.56)$ \\
Yes & 30 & & \\
No & & 11730 & $0.222(0.151-0.326)$ \\
Roof type $(P=0.077)$ & 26 & 7531 & $0.359(0.246-0.523)$ \\
Corrugated iron & 27 & & \\
Grass & &
\end{tabular}

aper 100 child-weeks-at-risk.

were significantly lower than those in children visited weekly, assuming each weekly visit represented 1 day's time at risk (Table 4). All children with a measured fever also gave a history of fever in the preceding $24 \mathrm{~h}$. The incidence of attendance to PCD was slightly higher in children visited daily than in those visited weekly (Table 4).

\section{Discussion}

The Ifakara study area is a heterogeneous setting largely consisting of simple dwellings made of mud bricks with earth floors and grass roofs. Although the study site is centred on Ifakara town the area can at best be described as semi-urban and there is little to distinguish the outskirts of the study area from the surrounding villages with high documented EIRs. However, the EIR of the Ifakara study area was recently estimated at 29 infective bites per person per year (Drakeley et al., 2003) and, in the current study, only $8 \%$ of children had a malaria episode detected during the year of follow-up. There was no clear age pattern of clinical disease and the maximum parasite prevalence was only $25 \%$, reached in children aged 4 years. Although there was significant month-to-month variation in the inci- 


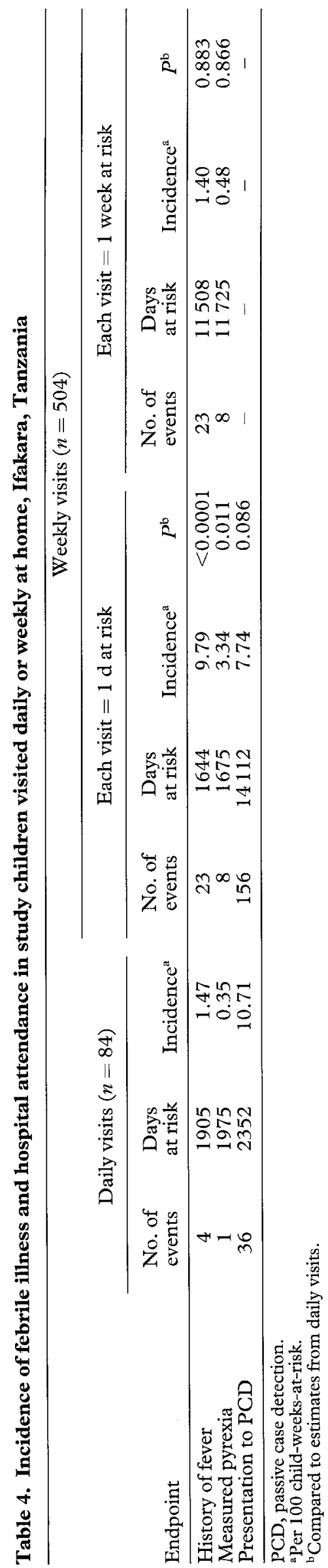

dence of clinical malaria there were incident cases in all but 1 month. That the intensity of transmission was sufficient to induce some immunity is suggested by the observations that none of the parasitaemias documented through the cross-sectional survey were associated with fever and that the peak parasite prevalence occurred 2 months after the peak in incident malaria cases. However, the incidence of clinical malaria was similar in infants and older children, suggesting the rate at which immunity develops is slow, consistent with the low $P$. falciparum challenge. Taken together, these findings show that the Ifakara study area is one of low-tomoderate, perennial $P$. falciparum transmission.

Why was the intensity of malaria transmission so much lower in Ifakara than in the surrounding villages? No intervention was delivered through the project, although study children had good access to curative services and this may have influenced the incidence of clinical malaria detected. Our results suggest some other possible explanations. In addition to month of the year, the incidence of clinical malaria was significantly associated with reported use of mosquito nets and the distance between the child's home and the hospital. The protective antimalarial effect of mosquito nets has been well documented (Lengeler, 2003) and $61 \%$ of our study children slept under a mosquito net the night before recruitment to the study. The increasing risk of clinical malaria with increasing distance between the residence and SFDDH has been observed in studies detecting malaria episodes by PCD (Acosta et al., 1999; Schellenberg et al., 2001). It was not clear whether this association was due to differences in health-seeking behaviour, due to reduced availability of alternative malaria treatment outlets further from SFDDH, or whether exposure to $P$. falciparum increased with increasing distance from SFDDH. By using ACD the current study eliminated differences in health-seeking behaviour and this association is therefore most likely to be a function of increasing proximity of the households to mosquito breeding sites. The nonsignificant tendency for roof type to be associated with risk of malaria may reflect a predisposition of Anopheles mosquitoes for grass roofs over iron roofs as they are cooler, have more potential resting places and afford easier access through incompletely sealed eaves (Lindsay \& Snow, 1988). However, this observation may also be explained by confounding associations between building materials and risk of malaria with socio-economic status or proximity to breeding sites.

Malaria is known to cause about $60 \%$ of the severe anaemia in infants in the study area (Menendez et al., 1997) and the current study offers some insights into the mechanisms of malaria-related anaemia. There was markedly less severe anaemia than that seen in a large community-based cross-sectional survey in 4 districts in southern Tanzania (Schellenberg et al., 2003), and only a non-significant decrease in the prevalence of anaemia with age. These 2 observations are consistent with the lower incidence of malaria in Ifakara than in the surrounding area, and may also reflect good access to curative services in the study area. The latter may also explain why the lowest prevalence of anaemia occurred a month after the peak incidence of symptomatic clinical malaria, when children would have had a haematological response to the treatment given. The importance of asymptomatic $P$. falciparum parasitaemia in the pathogenesis of anaemia is suggested by the maximal prevalence of asymptomatic parasitaemia being followed a month later by a peak in the prevalence of anaemia. However, these patterns should be interpreted with caution as several of the associations did not reach statistical significance.

Overall, only $26 \%$ of children with a history of fever or measured pyrexia were parasitaemic, underlining the poor specificity of fever as a marker for malaria. Furthermore, the marked age-dependence of fever, but 
lack of age-dependence of clinical malaria, indicates a higher incidence of non-malaria febrile illnesses in infants than in older children. Co-morbidity may therefore be more likely in infants than in older children and may result in the age pattern of malaria morbidity being different from the age pattern of malaria-associated mortality. The change with age of the fraction of fevers attributable to malaria may justify the use of different malaria case definitions in different age groups.

It has been suggested that PCD may tend to detect more serious malaria episodes than ACD (Trape \& Rogier, 1995). However the geometric mean parasite density in cases detected by PCD was similar to that in cases detected by ACD, although the relevance of parasitaemia as a marker of severity has been questioned in this setting (Schellenberg et al., 1999). Nevertheless, the similarities in the patterns seen in ACD and PCD of parasite densities and the association between location of house and risk of malaria reassure that PCD in Ifakara is not biased by health seeking or geographical factors.

Classically, the time at risk within an ACD study starts at recruitment and ends when follow-up is complete, implying that weekly visits each contribute $7 \mathrm{~d}$ to the time at risk. However, it is inevitable that intermittent visits will miss a proportion of fever episodes and the incidence of disease will be underestimated. An alternative approach is to consider each visit as representing 1 day's time at risk, increasing the estimated incidence rates by a factor of 7 . One would expect this to yield an incidence estimate similar to that obtained by visiting children on a daily basis. We were surprised, therefore, when daily visits to a random sample of the cohort generated incidence estimates substantially lower than those in which each weekly visit contributed one day to the time at risk. This may partly have resulted from individual febrile episodes each lasting several days, increasing the chance of their being detected by a weekly visit, and thus increasing the incidence of disease estimated by weekly visits. However, this would not explain the increased tendency of children visited daily to be brought to curative services at PCD. The children selected for daily visits were similar to the remainder of the cohort in those respects known to alter risk of illness, suggesting that the difference in incidence rates was largely due to the daily visits themselves, a so-called Hawthorne effect (Last, 1995). A possible explanation is that mothers of children visited more frequently developed a heightened awareness of their child's welfare and changed their caring patterns. This raises questions about the effect of weekly visits on estimated incidence rates, compared to no visits. An indication of this may be discerned from a comparison with infants from the same area involved in a contemporaneous intervention study (Schellenberg et al., 2001). In that study, the incidence of clinical malaria episodes, detected by PCD only, was 0.42 episodes per infant per year, which compares with the 0.13 episodes per infant per year in the current study. This further supports the suggestion that visiting children at home by ACD greatly influences the estimate of incidence rates and reveals that ACD in Ifakara is less likely than PCD to give an accurate estimate of the true incidence of malaria. However, the size and direction of this Hawthorne effect may vary in different age groups and in different settings. A review of data from The Gambia suggests a significant increase in the incidence of malaria detected in children visited weekly compared to those visited monthly (Greenwood et al., 1987) and a trend towards more episodes detected by daily compared to weekly visits (Snow et al., 1989). For these reasons, and because of differences in study design and case definitions, more weight should be placed on the age pattern of disease than on the absolute incidence rates detected by ACD in different sites.

A final methodological consideration concerns the malaria case definition. If it is accepted that each visit should represent 1 day's time at risk then it makes sense to include a history of fever in the preceding $24 \mathrm{~h}$ in the malaria case definition, and not just measured pyrexia. Although this will tend to reduce the specificity of the case definition it will increase its sensitivity and enhance the robustness of the rationale on which disease incidence rates are calculated.

In conclusion, the semi-urban Ifakara study area is subject to low-to-moderate perennial malaria transmission. The reduced intensity of transmission in comparison with the surrounding area is likely to be due to a combination of mosquito net use, reduced numbers of mosquito breeding sites in the centre of the study area and the better access to curative services. Including a history of fever in the malaria case definition may be rational when each $A C D$ visit contributes $1 \mathrm{~d}$ to the time at risk. ACD visits appear to be associated with a marked Hawthorne effect, making the age-pattern of disease detected through ACD a more robust indicator of disease epidemiology than absolute disease incidence estimates.

\section{Acknowledgements}

This study would not have been possible without the goodwill and cooperation of the families of study children and their community leaders. We are also grateful to the Kilombero District Health Management Team, SFDDH and IHRDC staff for general support. 'This investigation received financial support from the Fondo de Investigaciones Sanitarias (FIS number 00/0803) and the UNDP/World Bank/WHO Special Programme for Research and Training in Tropical Diseases (TDR).

\section{References}

Acosta, C. J., Galindo, C. M., Schellenberg, D., Aponte, J. J., Kahigwa, E., Urassa, H., Schellenberg, J. R., Masanja, H., Hayes, R., Kitua, A. Y., Lwilla, F., Mshinda, H., Menendez, C., Tanner, M. \& Alonso, P. L. (1999). Evaluation of the SPf66 vaccine for malaria control when delivered through the EPI scheme in Tanzania. Tropical Medicine and International Health, 4, 368-376.

Alonso, P. L., Smith, T., Schellenberg, J. R., Masanja, H., Mwankusye, S., Urassa, H., Bastos de Azevedo, I., Chongela. J., Kobero, S., Menendez, C., Hurt, N., Thomas, M., Lyimo, E., Weiss, N., Hayes, R., Kitua, A., Lopez, M., Kilama, W., Teuscher, T. \& Tanner, M. (1994). Randomised trial of efficacy of SPf66 vaccine against Plasmodium falciparum malaria in children in southern Tanzania. Lancet, 344, 1175-1181.

Armstrong Schellenberg, J. R. M., Greenwood, B. M., Gomez, P., Menendez, C. \& Alonso, P. L. (1994). Diurnal variation in body temperature of Gambian children. Transactions of the Royal Society of Tropical Medicine and Hygiene, 88, 429-431.

Armstrong Schellenberg, J., Mukasa, O., Abdulla, S., Marchant, T., Lengeler, C., Kikumbih, N., Mshinda, H. \& Nathan, R. (2001). The Ifakara Demographic Surveillance System. In: INDEPTH Monograph Series: Demographic Surveillance Systems for Assessing Populations and their Health in Developing Countries, Volume 1: Population, Health and Survival in INDEPTH Sites. Ottawa: International Development Research Centre, pp. 159-164.

Charlwood, J. D., Smith, T., Lyimo, E., Kitua, A. Y., Masanja, H., Booth, M., Alonso, P. L. \& Tanner, M. (1998). Incidence of Plasmodium falciparum infection in infants in relation to exposure to sporozoite-infected anophelines. American Fournal of Tropical Medicine and Hygiene, 59, 243-251.

Drakeley, C., Schellenberg, D., Kihonda, J., Sousa, C. A., Arez, A. P., Lopes, D., Lines, J., Mshinda, H., Lengeler, C., Armstrong Schellenberg, J., Tanner, M. \& Alonso, P. (2003). An estimation of the entomological inoculation rate for Ifakara: a semi-urban area in a region of intense malaria transmission in Tanzania. Tropical Medicine and International Health, 8, 767-774.

Greenwood, B. M., Bradley, A. K., Greenwood, A. M., Byass, P., Jammeh, K., Marsh, K., Tulloch, S., Oldfield, F. S. J. \& Hayes, R. (1987). Mortality and morbidity from malaria among children in a rural area of The Gambia, West Africa. Transactions of the Royal Society of Tropical Medicine and Hygiene, 81, 478-486. 
Hatz, C., Abdullah, S., Mull, R., Schellenberg, D., Gathmann, I., Kibatala, P., Beck, H.-P., Tanner, M. \& Royce, C. (1998). Efficacy and safety of CGP 56697 (artemether and benflumetol) compared with chloroquine to treat acute falciparum malaria in Tanzanian children aged $1-5$ years. Tropical Medicine and International Health, 3, 498-504.

Last, J. M. (1995). A Dictionary of Epidemiology, 3rd edition. Oxford: Oxford University Press.

Lengeler, C. (2003). Insecticide-treated bednets and curtains for preventing malaria (a Cochrane Review). In: The Cochrane Library, issue 4, 2003. Chichester: John Wiley \& Sons, Cochrane Database Systematic Review, CD000363.

Lindsay, S. W. \& Snow, R. W. (1988). The trouble with eaves; house entry by vectors of malaria. Transactions of the Royal Society of Tropical Medicine and Hygiene, 82, 645-646.

Lyimo, E. (1993). Some aspects of the adult bionomics of Anopheles gambiae s.1. in relation to malaria transmission in southeastern Tanzania. PhD thesis, Wageningen Agricultural University, The Netherlands.

Menendez, C., Kahigwa, E., Hirt, R., Vounatsou, P., Aponte, J. J., Font, F., Acosta, C. J., Schellenberg, D. M., Galindo, C. M., Kimario, J., Urassa, H., Brabin, B., Smith, T. A., Kitua, A. Y., Tanner, M. \& Alonso, P. L. (1997). Randomised placebo controlled trial of iron supplementation and malaria chemoprophylaxis for prevention of severe anaemia and malaria in Tanzanian infants. Lancet, 350, 844-850.

Schellenberg, D., Menendez, C., Kahigwa, E., Font, F., Galindo, C., Acosta, C., Armstrong Schellenberg, J., Aponte, J. J., Kimario, J., Urassa, H., Mshinda, H., Tanner, M. \& Alonso, P. (1999). African children with malaria in an area of intense Plasmodium falciparm transmission: features on admission to the hospital and risk factors for death. American fournal of Tropical Medicine and Hygiene, 61, $431-438$.

Schellenberg, D., Menendez, C., Kahigwa, E., Aponte, J., Vidal, J., Tanner, M., Mshinda, H. \& Alonso, P. (2001). Intermittent treatment for malaria and anaemia control at time of routine vaccinations in Tanzanian infants: a randomised, placebo-controlled trial. Lancet, 357, 1471-1477.

Schellenberg, D., Kahigwa, E., Drakeley, C., Malende, A. Wigayi, J., Msokame, C., Aponte, J. J., Tanner, M. Mshinda, H., Menendez, C. \& Alonso, P. L. (2002). The safety and efficacy of sulfadoxine-pyrimethamine, amodiaquine and their combination in the treatment of uncomplicated Plasmodium falcipamm malaria. American foumal of Tropical Medicine and Hygiene, 67, 17-23.

Schellenberg, D., Armstrong Schellenberg, J., Mushi, A., de Savigny, D., Mgalula, L., Mbuya, C. \& Victora, C. (2003). The silent burden of anaemia in Tanzanian children: a community-based study. Bulletin of the World Health Organization, 81, 581-590.

Sinnott, R. (1984). Virtues of the Haversine. Sky and Telescope, 68, 159

Smith, T., Charlwood, J. D., Kihonda, J., Mwankusye, S., Billingsley, P., Meuwissen, J., Lyimo, E., Takken, W. Teuscher, T. \& Tanner, M. (1993). Absence of seasonal variation in malaria parasitaemia in an area of intense seasonal transmission. Acta Tropica, 54, 55-72.

Snow, R. W., Menon, A. \& Greenwood, B. M. (1989). Measuring morbidity from malaria. Annals of Tropical Medicine and Parasitology, 83, 321-323.

Tanner, M., Del Guidice, G., Betschart, B., Biro, S., Burnier, E., Degrémont, A., Engers, H., Freyvogel, T., Lambert, P., Pessi, A., Speiser, F., Verdini, A. \& Weiss, N. (1986). Malaria transmission and development of anti-sporozoite antibodies in a rural African community. Memorias do Instituto Oswaldo Cruz, 86, supplement II, 199-205.

Trape, J. F. \& Rogier, C. (1995). Efficacy of SPf66 vaccine against Plasmodium falciparum malaria in children. Lancet, $345,134-135$.

Received 3 December 2002; revised 30 May 2003; accepted for publication 5 fune 2003 